\title{
Erratum to: Mannose-binding lectin- associated serine protease 2 (MASP-2) contributes to poor disease outcome in humans and mice with pneumococcal meningitis
}

\author{
E. Soemirien Kasanmoentalib ${ }^{1}$, Mercedes Valls Seron ${ }^{1}$, Bart Ferwerda ${ }^{1}$, Michael W. Tanck ${ }^{2}$, Aeilko H. Zwinderman², \\ Frank Baas ${ }^{3}$, Arie van der Ende ${ }^{4,5}$, William J. Schwaeble ${ }^{6 *}$, Matthijs C. Brouwer ${ }^{1}$ and Diederik van de Beek ${ }^{1,7^{*}}$
}

\section{Erratum}

Upon publication of the original article [1], it was noticed that Prof William J Schwaeble was omitted from the author list. He has now been added in this erratum, along with his affiliation and email address, as a co-corresponding author. Consequently, the numbering of Diederik van de Beek's affiliation ' 6 ' has been shifted to ' 7 '.

The new article reference should read:

Kasanmoentalib ES, Valls Seron M, Ferwerda B, Tanck MW, Zwinderman AH, Baas F, van der Ende A, Schwaeble WJ, Brouwer MC, van de Beek D. Mannose-binding lectin-associated serine protease 2 (MASP-2) contributes to poor disease outcome in humans and mice with pneumococcal meningitis. J Neuroinflammation. 2017 Jan $3 ; 14(1): 2$

\footnotetext{
Author details

'Department of Neurology, Academic Medical Center, Amsterdam Neuroscience, Amsterdam, The Netherlands. ${ }^{2}$ Department of Clinical Epidemiology, Biostatistics, and Bioinformatics, Academic Medical Center, University of Amsterdam, Amsterdam, The Netherlands. ${ }^{3}$ Department of Genome Analysis, Academic Medical Center, Amsterdam, The Netherlands. ${ }^{4}$ Department of Medical Microbiology, Center of Infection and Immunity Amsterdam (CINIMA), Academic Medical Center, Amsterdam, The Netherlands. ${ }^{5}$ The Netherlands Reference Laboratory for Bacterial Meningitis, Center of Infection and Immunity Amsterdam (CINIMA), Academic Medical Center, Amsterdam, The Netherlands. ${ }^{6}$ Department of Infection, Immunity and Inflammation, University of Leicester, Leicester, UK. ${ }^{7}$ Department of Neurology, Academic Medical Center, University of Amsterdam, Amsterdam Neuroscience, PO Box 226601100 DD Amsterdam, The Netherlands.
}

Received: 29 March 2017 Accepted: 29 March 2017

Published online: 06 April 2017

\section{Reference}

1. Kasanmoentalib ES, Valls Seron M, Ferwerda B, Tanck MW, Zwinderman AH, Baas F, van der Ende A, Brouwer MC, van de Beek D. Mannose-binding lectin-associated serine protease 2 (MASP-2) contributes to poor disease outcome in humans and mice with pneumococcal meningitis. J Neuroinflammation. 2017;14:9.

*Correspondence: ws5@le.ac.uk; d.vandebeek@amc.nl

${ }^{6}$ Department of Infection, Immunity and Inflammation, University of Leicester, Leicester, UK

'Department of Neurology, Academic Medical Center, Amsterdam

Neuroscience, Amsterdam, The Netherlands 